\title{
Syphilitic Hepatitis: An Uncommon Manifestation
}

\author{
Leila Amaro Cardoso ${ }^{\text {a e e, f }}$, Ruben Melo Carvalho ${ }^{\text {b, e }}$, Rui Caetano Oliveira ${ }^{c}$, \\ Ana Gameiro ${ }^{\text {d }}$, Eduardo Rabadao ${ }^{\mathrm{b}}$, Jose Saraiva da Cunha ${ }^{\mathrm{b}}$, \\ Armando Carvalho ${ }^{\text {a }}$
}

\begin{abstract}
Syphilis is a systemic disease caused by the transmission through sexual contact of Treponema pallidum. It has a high incidence in men who have sex with other men, and is associated with human immunodeficiency virus (HIV) infection. The diagnosis depends on serological tests or confirmation of the spirochete in histological samples. The primary lesion courses after a latency period of 2 to 6 weeks, and the secondary disseminated phase, usually associated with mucocutaneous lesions and lymphadenopathy, may involve any organ. Symptomatic syphilitic hepatitis is a rare manifestation, the diagnosis of which is a challenge. It is characterized by a marked elevation of alkaline phosphatase, moderate elevation of transaminases, and no bilirubin changes. Histologically, portal inflammation and non-caseous granulomas predominate without associated necrosis. The case described is a rare manifestation of secondary syphilis, in the form of hepatitis in a sexually active HIV-infected patient with good response to the recommended benzathine penicillin treatment.
\end{abstract}

Keywords: Syphilis; Treponema pallidum; Hepatitis; Maculopapular rash

\section{Introduction}

Syphilis is a complex systemic disease caused by Treponema pallidum, a very invasive spirochete. It is transmitted through sexual contact, with a high incidence in men who have sex with men (MSM), and is associated with other sexually trans-

Manuscript submitted January 10, 2019, accepted February 1, 2019

aServico de Medicina Interna do Centro Hospitalar Universitario de Coimbra, Coimbra, Portugal

bServico de Infecciologia do Centro Hospitalar Universitario de Coimbra, Coimbra, Portugal

'Servico de Anatomia Patologica do Centro Hospitalar Universitario de Coimbra, Coimbra, Portugal

dServico de Dermatologia do Centro Hospitalar Universitario de Coimbra, Coimbra, Portugal

${ }^{\mathrm{e}}$ Contributed equually

${ }^{f}$ Corresponding Author: Leila Amaro Cardoso, Servico de Medicina Interna B, Hospital Geral do Centro Hospitalar Universitario de Coimbra, 3046-853 Coimbra, Portugal. Email: leilaamarocardoso@gmail.com

doi: https://doi.org/10.14740/jmc3254 mitted diseases (STDs) such as human immunodeficiency virus (HIV) infection [1]. Diagnosis is not made by isolation in culture, but usually depends on serological tests or confirmation of the spirochete in histological samples [2].

It occurs with primary lesion after a latency period of 2 to 6 weeks. The secondary phase, disseminated, usually associated with mucocutaneous lesions and lymphadenopathy, may involve any organ [3].

Symptomatic syphilitic hepatitis is a rare manifestation, characterized by a marked elevation of alkaline phosphatase, moderate elevation of transaminases, and no changes in bilirubin. Histologically, portal inflammation and non-caseous granulomas are identified without associated necrosis [4].

The treatment involves the use of benzathine penicillin, with the schedule depending on the stage of the disease and the organs involved [5].

\section{Case Report}

A 46-year-old male, leukodermal, MSM, had been followed by a diagnosis of infection with HIV for 5 years, with sustained virological suppression and cluster of differentiation (CD)4+ $\mathrm{T}$ lymphocyte count of $516 / \mathrm{mm}^{3}$, under antiretroviral therapy with tenofovir, emtricitabine and efavirenz. The patient had a history of unprotected sexual contacts with multiple partners in previous years.

He was admitted by pruritic maculopapular rash localizing to the dorsal region and the proximal region of the lower limbs, with changes in the evidence of hepatic function. Abdominal objective examination showed no significant changes, such as organomegaly. There was no symptom or signs at the genital level.

From the analytical study, a mixed pattern hepatitis of predominant cholestatic predominance with alkaline phosphatase (308 U/L), with infection screening for hepatitis A, B, C and E viruses was negative.

IgG/IgM serology (CLIA) was positive, rapid plasma reagin (RPR) of 512 and fluorescent treponemal antibody-absorption (FTA-ABS) positive.

Based on the laboratory findings, biopsy, cutaneous and hepatic biopsies were performed, with primary suspicion of secondary lesions of syphilitic disease.

Cutaneous biopsy (Fig. 1) showed mild inflammatory infiltrate by lymphomonuclear cells, occasional dispersed eosinophils and neutrophils, some of which were intravascular, changes compatible with syphilitic involvement. 


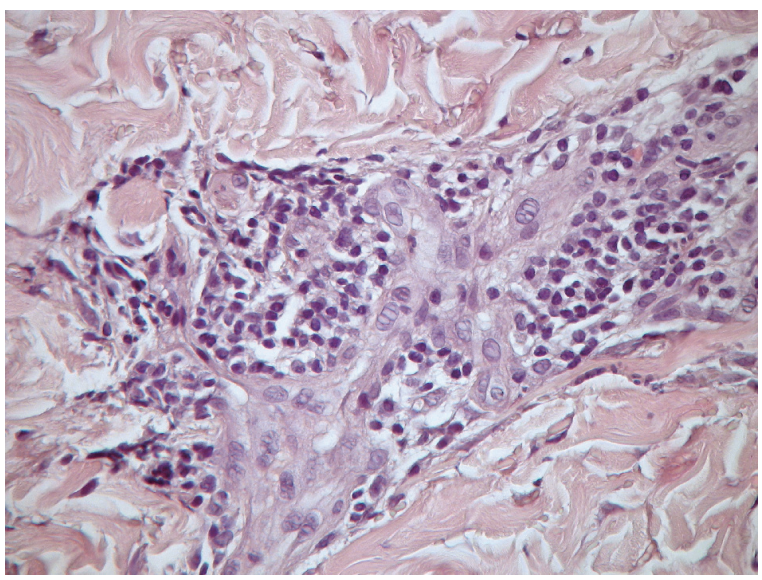

Figure 1. H\&E stain, inflammatory infiltrate by lymphomonuclear cells, dispersed eosinophils and neutrophils, some of which are intravascular.

Hepatic biopsy was performed (Fig. 2), with description of mononuclear inflammatory infiltrate of some portal spaces and discrete lobular necroinflammatory activity, with dispersed acidophilic bodies and focal formation of epithelioid granulomatous aggregates without necrosis compatible with hepatic involvement by syphilis.

The patient was treated with three doses per week of 2.4 million units of benzathine penicillin for a total of 7.2 million units and was asymptomatic with resolution of rash and cutaneous pruritus. Standardization of the transaminases and enzymes of hepatic cholestasis was verified.

\section{Discussion}

Secondary syphilis can affect any organ, sometimes hindering and delaying diagnosis, particularly when there is no cutaneous involvement. The fact that our patient has secondary lesions with maculopapular rash and hepatic cholestasis without other probable cause has increased our suspicion of syphilitic hepatitis.

As described in the literature, most patients with hepatic involvement are asymptomatic, $50 \%$ of whom have only slight elevation of alkaline phosphatase [4]. In rare cases with symptomatic hepatitis, there is a particular increase in cholestatic enzymes. As seen in our histological piece, there is portal inflammation, without hepatocellular necrosis and non-caseous granulomas.

The usefulness of this case is the description of a rare manifestation of secondary syphilis in the form of hepatitis, with good response to the recommended treatment with benzathine penicillin. In HIV-infected patients, sexually active, this should be one of the differential diagnoses to be taken into account.

\section{Acknowledgments}

None to declare.

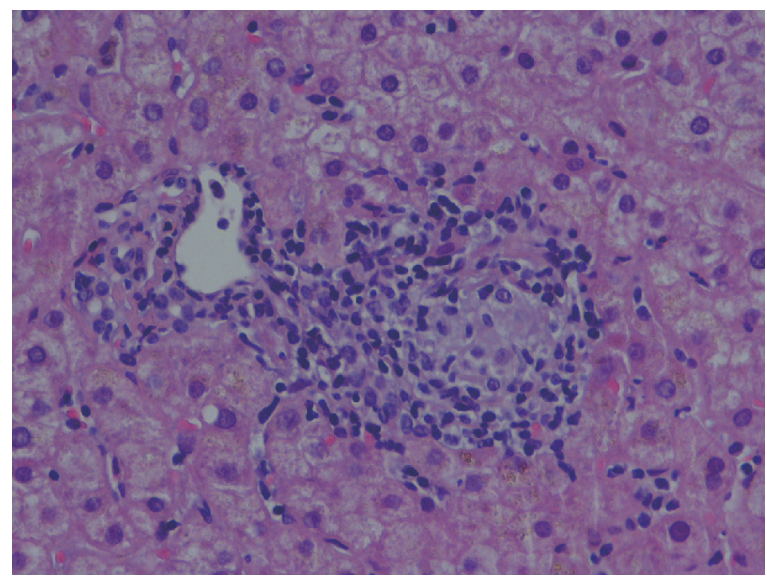

Figure 2. H\&E stain, epithelioid granuloma in portal space, not necrotizing.

\section{Financial Disclosure}

None to declare.

\section{Conflict of Interest}

None to declare.

\section{Informed Consent}

Informed consent was obtained from the patient.

\section{Author Contributions}

Leila Cardoso and Ruben Carvalho contributed to the elaboration, Eduardo Rabadao, Jose Saraiva and Armando Carvalho contributed to the review, Ana Gameiro contributed in the cutaneous biopsy, Rui Caetano Oliveira contributed in the hepatic biopsy.

\section{References}

1. Ghanem KG, Workowski KA. Management of adult syphilis. Clin Infect Dis. 2011;53(Suppl 3):S110-128.

2. Cantor AG, Pappas M, Daeges M, Nelson HD. Screening for syphilis: updated evidence report and systematic review for the US Preventive Services Task Force. JAMA. 2016;315(21):2328-2337.

3. Chapel TA. The signs and symptoms of secondary syphilis. Sex Transm Dis. 1980;7(4):161-164.

4. Hussain N, Igbinedion SO, Diaz R, Alexander JS, Boktor M, Knowles K. Liver cholestasis secondary to syphilis in an immunocompetent patient. Case Reports Hepatol. 2018;2018:8645068.

5. Clement ME, Okeke NL, Hicks CB. Treatment of syphilis: a systematic review. JAMA. 2014;312(18):1905-1917. 